\title{
Conocimientos y actitudes hacia la donación de sangre en una población universitaria de Chile
}

\author{
Marcela Vásquez, ${ }^{1}$ Patricia Ibarra ${ }^{1}$ y Mónica Maldonado ${ }^{1}$
}

Forma de citar

Vásquez M, Ibarra P, Maldonado M. Conocimientos y actitudes hacia la donación de sangre en una población universitaria de Chile. Rev Panam Salud Publica. 2007;22(5):323-8.

RESUMEN Objetivos. Evaluar los conocimientos y actitudes hacia la donación de sangre voluntaria entre estudiantes, académicos y no académicos de la Universidad de Talca, Chile.

Métodos. Entre junio y julio de 2002, se aplicó una encuesta, previamente validada, a 487 personas de la comunidad universitaria, para indagar acerca de su percepción con respecto a algunos aspectos de la donación de sangre, tales como: conocimientos y motivaciones sobre la donación, y desmotivaciones y mitos asociados a la donación de sangre. Para el análisis de los datos se usaron estadígrafos descriptivos.

Resultados. De los encuestados sólo 14\% habían donado sangre y correspondían mayoritariamente al grupo de académicos (43,3\%). Los estudiantes fueron quienes, en mayor medida, manifestaron su intención de donar sangre en el futuro $(88,1 \%)$. Al comparar la predisposición a donar sangre en el futuro entre hombres y mujeres, se obtuvieron porcentajes de $90 \%$ y $84,2 \%$ respectivamente; sin embargo, esta diferencia no fue estadísticamente significativa. Dentro de las desmotivaciones para donar sangre, 73,4\% de los encuestados dijo desconfiar de la esterilidad del material empleado en la colección de sangre.

Conclusiones. La población universitaria es un grupo muy deseable para convertirlo en donantes voluntarios; una adecuada campaña de información y motivación podría transformar a $87,3 \%$ de las personas con intención de donar sangre, en donantes voluntarios reales.

Palabras clave Donadores de sangre, instituciones de enseñanza superior, actitudes, Chile.

Al inicio de los años noventa se hizo un diagnóstico de la situación de los bancos de sangre en Chile, que permitió constatar la existencia de 180 instituciones de ese tipo, de las cuales

\footnotetext{
1 Departamento de Bioquímica Clínica e Inmunohematología, Facultad de Ciencias de la Salud, Universidad de Talca, Chile. La correspondencia se debe enviar a Marcela Vásquez Rojas, Profesora, Facultad de Ciencias de la Salud, Universidad de Talca, 2 Norte No. 685, Talca, Chile. T: 5671-200494. F: 56-71-200488. Correo electrónico: mvasquro@utalca.cl
}

$68,5 \%$ eran públicas; tan sólo en $23 \%$ de los bancos de sangre se llevaban a cabo $90 \%$ de las transfusiones; había una eliminación por obsolescencia de $12 \%$ de los productos y un bajo índice de fraccionamiento; asimismo, se comprobó la falta de control de calidad y que $98 \%$ de las donaciones de sangre se realizaban a través del sistema intrafamiliar o de reposición (1).

Los resultados de este diagnóstico motivaron a las autoridades a estudiar la factibilidad de centralizar la pro- ducción de hemocomponentes, tal como se hace en países industrializados como Inglaterra, Francia y España, entre otros (2). De esta forma, se aprobó un proyecto que plantea la centralización en cuatro sitios productores que deberían atender las necesidades de sangre de las unidades de medicina transfusional de cada hospital. Este proyecto favorecería la equidad, calidad, oportunidad y eficiencia de la medicina transfusional en Chile (1). 
Uno de los pilares fundamentales para el funcionamiento de un sistema centralizado es la donación de sangre altruista y, en la medida de lo posible, a repetición, es decir, personas que donan sangre a otras que la necesitan, y que lo hacen con el único fin de servir a la humanidad sin buscar retribución alguna. Cabe destacar que en Chile las cifras indicaban que en el año 2000 los donantes de sangre altruistas representaban únicamente 2,6\% del total de donaciones anuales.

Existen dos grandes debilidades en el sistema de donantes de sangre de reposición: por un lado, la alta prevalencia de patógenos de transmisión sanguínea y, por el otro, el cuestionamiento ético que surge de la "obligatoriedad" de este sistema. En el plano internacional, se ha demostrado que la donación voluntaria y repetida de sangre hace de la transfusión sanguínea un proceso más seguro, por la menor tasa de transmisión de agentes infecciosos vinculados con la transfusión (3-5).

Para implementar estrategias tendentes a incrementar la donación, es necesario iniciar el proceso recabando información, en la comunidad respectiva, acerca de sus actitudes y motivaciones hacia la donación de sangre, así como sobre los conocimientos que tiene sobre la sangre, la donación y la utilización. Se han realizado diversos estudios en este sentido, en población hispana de la localidad de Rio Grande Valley, en el estado de Texas, en los Estados Unidos de América, donde se determinó que las personas con un mayor nivel de educación, que hablasen inglés y pertenecieran a un nivel socioeconómico más elevado, tendrían más probabilidades de donar sangre. Los resultados de otros dos trabajos fueron congruentes con lo anterior, pues se encontró que las personas con un menor nivel de educación tienen menos probabilidades de donar sangre, en comparación con aquellas que poseen un nivel de instrucción más alto (6-8). Por su parte, a fines de 1999 la Organización Panamericana de la Salud (OPS) expresó, en la resolución CD4 R15/9 (3), la necesidad de que los "Programas Nacionales de Sangre y Servicios de Transfusión, tuviesen como base la donación altruista y repetida de sangre".

De todo lo señalado previamente, se desprende la urgencia de incrementar los esfuerzos para aumentar el porcentaje de donación de sangre altruista. Para lograr esto, es necesario elaborar un diagnóstico que permita saber acerca de las percepciones y conocimientos de la población local, con relación a este tema. A partir de los datos que se obtengan, podrá incrementarse la información necesaria para estructurar las campañas y estrategias de promoción de la donación de sangre altruista.

\section{MATERIALES Y MÉTODOS}

Población. El estudio de tipo descriptivo, transversal, se desarrolló en junio y julio de 2002 en los campus Lircay y Los Niches ${ }^{2}$ de la Universidad de Talca, en Chile, con una población de 4800 personas agrupadas en 4300 estudiantes, 270 académicos y 230 funcionarios no académicos. La muestra definida, correspondiente a 500 personas, se estratificó de acuerdo con los grupos indicados anteriormente; para el estrato estudiantes se consideró una subestratificación proporcional por carreras, a fin de garantizar que todas estuviesen representadas. El muestreo se realizó por conveniencia, para el caso de los estudiantes, mediante contacto con los directores de cada escuela y reclutando al número predefinido de alumnos en las clases a cargo de ese profesor. Para el caso de los académicos y no académicos, el muestreo se realizó mediante invitación en sus lugares de trabajo. Del total de encuestas tomadas, hubo 13 documentos que no pudieron incorporarse al análisis por estar incompletos, de tal manera que la muestra final quedó conformada por 487 personas.

\footnotetext{
En 2002 la Universidad de Talca tenía dos campus con 13 carreras de pregrado. En el campus Lircay se impartían las licenciaturas correspondientes a las facultades de ciencias: Jurídicas y Sociales; Forestales; Agrarias; Empresariales y de la Salud, mientras que en el campus Los Niches funcionaba la Facultad de Ingeniería; en este campus radicaba sólo $19 \%$ de la población universitaria total.
}

Encuesta. Para la recolección de los datos se aplicó una encuesta que incluía preguntas sobre características demográficas como sexo, edad, actividad y estado civil, junto a las 13 preguntas orientadas a detectar la percepción y conocimientos de los encuestados con respecto a la donación de sangre. Las preguntas se agruparon en cuatro conglomerados: I) Conocimientos sobre la donación de sangre; II) Motivación de las personas para donar sangre; III) Razones que desmotivan a las personas a donar, y IV) Mitos asociados a la donación de sangre. Las escalas empleadas para medir las variables fueron de tipo nominal y ordinal (anexo 1). La encuesta fue autoadministrada con posterioridad a una explicación dada por uno de los investigadores, para el caso de los estudiantes, al grupo reunido en una sala de clases y, para el caso de académicos y no académicos, a grupos que no superaban las cuatro personas.

Validación del cuestionario. El cuestionario usado en este estudio se sometió a prueba con grupos de 10 personas representantes de cada uno de los estratos definidos anteriormente, además de cinco personas que tenían conocimientos sobre el tema: tres tecnólogos médicos de banco de sangre y dos estadísticos. Las preguntas fueron evaluadas como "Buena", "Regular" o "Mala" con respecto al conglomerado al cual pertenecían, de tal manera que la que tenía un mínimo de calificación de $90 \%$ se clasificó como "Buena"; por otra parte, se eliminaron las preguntas con más de 10\% de calificación "Mala".

Análisis estadístico. Los datos del estudio se analizaron descriptivamente, expresados en porcentaje por el tipo de pregunta utilizada. Las escalas empleadas en el cuestionario fueron de tipo nominal y ordinal, de tal manera que el análisis se realizó con base en frecuencias absolutas y relativas. Asimismo se aplicó análisis de $\chi^{2}$ para determinar la significancia estadística de algunos resultados, mediante la utilización del programa EpiInfo versión 3.3.2. 


\section{RESULTADOS}

De las 487 personas que respondieron toda la encuesta, $87,7 \%$ (427) representaban el estrato de estudiantes, $6,2 \%$ (30) el de académicos y 6,2\% (30) el de no académicos; $53,1 \%$ eran del sexo masculino y el rango de edad de $88,6 \%$ de la población fluctuaba entre los 17 y 30 años y 89,6\% eran solteros (cuadro 1).

Conocimientos sobre la donación de sangre. Únicamente $14 \%$ de los encuestados afirmaron haber donado sangre, de los cuales $89,3 \%$ manifestaron tener conocimientos generales sobre las necesidades, utilización y análisis de la sangre y lugar de donación, aunque sólo 23,3 \% poseían conocimientos más específicos acerca de los requisitos de edad, peso e intervalos entre donaciones. Cabe hacer notar el hecho de que casi la mitad de los encuestados creía que se comercializaría la sangre $(49,6 \%)$.

Motivación para donar sangre. Con respecto a la predisposición hacia la donación de sangre, se encontró que del total de encuestados, 425 (87,3\%) estarían dispuestos a donar sangre en el futuro. El estrato de los estudiantes encabezó la lista con $88,1 \%$ de perso-

CUADRO 1. Características de la población seleccionada de la Universidad de Talca, Chile, junio-julio 2002

\begin{tabular}{lr}
\hline Característica & Frecuencia $(\%)$ \\
\hline Sexo & \\
Masculino & $259(53,1)$ \\
Femenino & $228(46,9)$ \\
Actividad & \\
$\quad$ Estudiante & $427(87,7)$ \\
Académico & $30(6,2)$ \\
No académico & $30(6,2)$ \\
Edad & \\
$17-30$ años & $431(88,6)$ \\
$31-40$ años & $20(4,1)$ \\
$41-50$ años & $22(4,5)$ \\
$>51$ años & $13(2,7)$ \\
Estado civil & \\
Soltero & $436(89,6)$ \\
Casado & $47(9,6)$ \\
Divorciado & $3(0,6)$ \\
Viudo & $1(0,2)$ \\
\hline
\end{tabular}

CUADRO 2. Distribución de frecuencia, por actividad, de posibles donadores de sangre. Universidad de Talca, Chile, junio-julio 2002

\begin{tabular}{lccc}
\hline \multicolumn{1}{c}{ Actividad } & \multicolumn{2}{c}{ Donaría sangre en el futuro } & $\begin{array}{c}\text { Total de } \\
\text { encuestados }\end{array}$ \\
\cline { 2 - 3 } Estudiantes & \multicolumn{1}{c}{$\%$} & $\%$ & 427 \\
No académicos & 376 & 88,1 & 30 \\
Académicos & 24 & 80,0 & 30 \\
Total & 25 & 83,3 & 487 \\
\hline
\end{tabular}

CUADRO 3. Distribución de frecuencia, por sexo, de posibles donadores de sangre. Universidad de Talca, Chile, junio-julio 2002

\begin{tabular}{lccc}
\hline Sexo & \multicolumn{2}{c}{ Donaría sangre en el futuro } & $\begin{array}{c}\text { Total de } \\
\text { encuestados }\end{array}$ \\
\cline { 2 - 3 } & $n$ & $\%$ & 259 \\
Hombres & 233 & 90,0 & 228 \\
Mujeres & 192 & 84,2 & 487 \\
Total & 425 & 87,3 & \\
\hline
\end{tabular}

Nota: No hubo diferencias estadísticamente significativas entre sexo $P>0,05$.

nas predispuestas, seguido de los académicos con $83,3 \%$ y de los no académicos, con $80 \%$, aunque estas diferencias no fueron estadísticamente significativas (cuadro 2). Al analizar la distribución por sexo, considerando el total de hombre y mujeres que participaron en el estudio, se observa que sólo 84,2\% (192/228) de las mujeres estarían dispuestas a donar sangre, en comparación con 90\% (233/259) de los hombres, pero estos porcentajes tampoco fueron estadísticamente significativos (cuadro 3).

Destacan como razones principales que motivan a donar sangre en el futuro el interés de ayudar a un familiar o a un amigo enfermo $(97,6 \%)$, o bien en el caso de una catástrofe nacional $(62,8 \%)$, ambas señaladas como muy importantes.

Al preguntar sobre el medio de comunicación por el cual les gustaría recibir información acerca de la donación, $62,3 \%$ de los encuestados prefirieron la televisión; la radio obtuvo el menor porcentaje $(3,6 \%)$ de preferencia. Por otra parte, a $4,7 \%$ de los encuestados no les interesaba recibir información alguna.
Razones que desmotivan a la donación sanguínea. Al sumar las valoraciones "Muy importante" y "Regularmente importante", las principales razones que desmotivan a los encuestados a donar son: las causas médicas $(75,7 \%)$; la desconfianza en la esterilidad del material $(73,4 \%)$, y haber tenido hepatitis $(64,2 \%)$. Por otra parte, también es notable el rechazo al ambiente hospitalario (48\%).

Mitos asociados a la donación de sangre. Del total de encuestados, sólo 7,8\% consideraban que donar sangre es dañino para la salud, y que las dos principales causas para esto son el riesgo de contraer alguna enfermedad (94,6\%) y la anemia (73\%), ambas catalogadas como razones muy importantes.

\section{DISCUSIÓN}

Cuatrocientas ochenta y siete personas de la Universidad de Talca, en Chile, respondieron satisfactoriamente una encuesta para indagar acerca del nivel de conocimiento y la percepción que tenían sobre la donación de san- 
gre. Más de $85 \%$ de los encuestados tenían menos de 30 años y eran solteros, lo que se explica por la alta proporción de estudiantes que conformaron la muestra en estudio. De las personas encuestadas, $86 \%$ nunca habían donado sangre, lo que concuerda con otros estudios realizados en poblaciones universitarias (9-11).

En relación con los conocimientos sobre la donación de sangre, se detectó que la población tiene información general sobre el tema, pero que carece de un manejo preciso de aspectos más específicos: sabe que la sangre es un recurso necesario y que se somete a análisis antes de ser utilizada, pero desconoce con qué frecuencia se puede donar, así como los requisitos y limitaciones para hacerlo. Estos datos son similares a los encontrados en universitarios de Bangladesh (10) y en la población representante de los 15 países de América que formaron parte del estudio que realizaran en 2003 García y colaboradores (3).

Por otro lado, más de $80 \%$ de la población encuestada tenía una actitud positiva hacia la donación de sangre y estaría dispuesta a hacerlo en el futuro. Esta tendencia también se ha detectado en una investigación efectuada en Inglaterra en 1991 (12), en estudiantes de Bangladesh (10) y de Tailandia (11), y donde se concluyó que la imagen que se tenía hacia la donación era muy positiva. Sin embargo, en el presente estudio se encontró que las razones que manifestó la población para donar serían la necesidad de un familiar o amigo enfermo, seguida de una catástrofe nacional. Esta es una actitud similar a la encontrada en otras personas de América, y una posible explicación sería que en estas regiones se piensa que la sangre es un recurso escaso y que, por ende, hay que reservar la donación para una situación específica. En la cultura latinoamericana está muy arraigada la idea de que sólo se debe donar sangre por motivos de fuerza mayor, y no hay conciencia de las necesidades permanentes de sangre (3). De esta forma, la actitud positiva no repercute en un incremento de donantes voluntarios.
La mayoría de las personas (95,3\%) estuvo de acuerdo en recibir información sobre la donación de sangre y $62,3 \%$ optaron por la televisión como medio de comunicación. Este dato debe considerarse en las estrategias de promoción, ya que la televisión es un medio masivo y de gran alcance, aunque de costo elevado. Estudios realizados en España señalan que para los no donantes, la mayor desmotivación es justamente la falta de información $(43,6 \%)(13,14)$. De lo anterior se desprende que deben diseñarse estrategias de comunicación social eficaces que contemplen información, educación y comunicación, orientadas a promover la donación voluntaria de sangre.

Al igual que en los trabajos de Thompson (6), Hosain y colaboradores (10) y Wiwanitkit (11), una de las razones más importantes que desmotivan a la donación sanguínea es la desconfianza hacia la esterilidad del material $\mathrm{y}$, por ende, el miedo a contagiarse de alguna enfermedad infecciosa como el SIDA. Dicha percepción se detectó también en población inglesa a inicios de los años noventa (15). Estos tabúes y mitos sobre la donación de sangre influyen negativamente en el reclutamiento de donantes voluntarios.

Por otra parte, se detectó una leve diferencia entre hombres $(90 \%)$ y mujeres $(84,2 \%)$ respecto de la actitud positiva hacia una futura donación de sangre. A pesar de que esa diferencia no fue estadísticamente significativa, sí concuerda con los resultados obtenidos en estudios de Boulware y colaboradores (16), quienes concluyeron que las mujeres están menos dispuestas a donar sangre, en este caso por el miedo que les ocasiona el ambiente hospitalario. Existen diversas razones que pueden explicar la tasa más baja de donación entre las mujeres $\mathrm{y}$, por ende, su menor predisposición a donar sangre, y que incluyen la preocupación por su salud con respecto a aspectos tales como la anemia, los embarazos, la pérdida de peso o el antecedente de haber sido rechazadas como donantes (12). En el presente estudio se podría asumir que razones similares justifican la menor predisposición a donar sangre detectada en mujeres considerando, además, que en la actualidad la proporción de mujeres que donan sangre en el sistema de reposición también es inferior respecto de los hombres. No obstante, la realización de estudios adicionales para conocer las causas específicas de este fenómeno permitiría detectar los aspectos potencialmente modificables en estos grupos y orientar de mejor forma las campañas de promoción de donación sanguínea.

Para promover la donación de sangre voluntaria, la OPS considera que, en cada país, deben trabajar activamente las instituciones académicas, las asociaciones profesionales, las sociedades científicas y los medios de comunicación, además de los bancos de sangre. Para que esto se logre, es indispensable terminar con la obligatoriedad de familiares y amigos de reponer la sangre transfundida, porque de lo contrario las poblaciones seguirán pensando en guardar el recurso sangre para las urgencias.

En resumen, los resultados del presente estudio permiten sugerir que las campañas de promoción de la donación voluntaria de sangre, en la población de la Universidad de Talca, deberían centrarse en la difusión de información más precisa sobre los requisitos para ser aceptado como donante de sangre, recalcando la seguridad del proceso desde el punto de vista del contagio de enfermedades infecciosas como el SIDA, e incorporando una motivación especial para las mujeres, haciendo énfasis en el hecho de que, en el proceso de selección de donantes, se realizan una entrevista y un examen físico para definir si la persona está o no en condiciones de hacer una donación.

Los aspectos relacionados con el efecto negativo que ejerce la coexistencia de la donación por reposición y la voluntaria sobre la predisposición a donar sangre, así como la necesidad de difusión masiva vía televisión, son cuestiones más compleja s de abordar, que requieren de una inversión económica y de cambios en las políticas. Los resultados de este estudio posiblemente podrían extrapolarse a otras universidades regionales y nacionales. 


\section{ANEXO 1. Cuestionario}

En Chile se pretende mejorar el abastecimiento de sangre para asegurar que quien necesite este vital elemento cuente con él en la cantidad suficiente y en el momento oportuno. Para lograr este propósito es importante hacer un diagnóstico de la actitud de la población ante la donación de sangre.

Sus respuestas y opinión son de gran importancia y de absoluta confidencialidad.

Instrucciones: Marque con una X la opción que represente mejor su opinión.

Sexo: $\quad M \square \quad F \square$

Edad: 17-30 años $\square \quad$ 31-40 años $\square \quad 41-50$ años $\square \quad>50$ años $\square$

Actividad: Estudiante

$\square$, carrera

Académico

$\square$, área

No académico

$\square$, área

Estado civil: Soltero $\square \quad$ Casado $\square \quad$ Otro:

1. ¿Ud. ha donado sangre alguna vez?

2. ¿Considera necesario donar sangre?

3. ¿Sabe para qué sirve la sangre donada?
Sí $\square \quad$ No $\square$

Sí $\square \quad$ No $\square$

Sí $\square \quad$ No $\square$
4. ¿Cuál es la edad mínima para donar?
a) 15 años
b) 18 años
c) Cualquier edad
d) No sabe

5. ¿Cuál es el peso mínimo para donar sangre?
a) 50 kilos
b) 60 kilos
c) Más de 80 kilos
d) No sabe

6. ¿Cuánto tiempo debe pasar entre una donación de sangre y otra?
a) 1 mes
b) 3 meses
c) 1 año
d) No sabe

7. ¿Cree Ud. que se comercializa con la sangre? Sí $\square$

No $\square$

8. ¿La sangre donada se analiza antes de ser $\quad$ Sí $\square \quad$ No $\square$ transfundida?

9. ¿Sabe dónde ir a donar sangre? Sí $\square \quad$ No $\square$

10. ¿Por qué medio de comunicación le gustaría recibir información acerca de la donación? (MARQUE SÓLO UNA):
a) T.V.
e) Correo
b) Radio
f) Internet
c) Periódico
g) No me interesa recibir información

11. De las siguientes razones para NO DONAR SANGRE, evalúe el nivel de importancia

\begin{tabular}{|c|c|c|c|c|c|}
\hline & $\begin{array}{c}\text { Muy } \\
\text { importante }\end{array}$ & $\begin{array}{c}\text { Reguralmente } \\
\text { importante }\end{array}$ & Neutral & $\begin{array}{c}\text { No tan } \\
\text { importante }\end{array}$ & $\begin{array}{l}\text { No es importante } \\
\text { en lo absoluto }\end{array}$ \\
\hline \multicolumn{6}{|l|}{ Por causas médicas } \\
\hline \multicolumn{6}{|c|}{ Desconfianza a la esterilidad del material } \\
\hline \multicolumn{6}{|c|}{ Por creer que se comercializa con la sangre } \\
\hline \multicolumn{6}{|l|}{ Por creencias religiosas } \\
\hline \multicolumn{6}{|c|}{ Por tener rechazo al ambiente hospitalario } \\
\hline \multicolumn{6}{|l|}{ Por haber tenido hepatitis } \\
\hline \multicolumn{6}{|l|}{ Por pesar poco } \\
\hline \multicolumn{6}{|l|}{ Por temor a la pérdida de sangre } \\
\hline Porque nadie me lo ha pedido & & & & & \\
\hline
\end{tabular}

12. ¿Cree que podría donar sangre en el futuro? $\quad$ Sí $\square \quad$ No $\square$

Sólo si su respuesta es "Sí", valore las siguientes razones por las cuales Ud. donaría sangre:

\begin{tabular}{|l|l|l|l|l|l|}
\hline & $\begin{array}{c}\text { Muy } \\
\text { importante }\end{array}$ & $\begin{array}{c}\text { Reguralmente } \\
\text { importante }\end{array}$ & Neutral & $\begin{array}{c}\text { No tan } \\
\text { importante }\end{array}$ & $\begin{array}{c}\text { No es importante } \\
\text { en lo absoluto }\end{array}$ \\
\hline Una catástrofe nacional & & & & \\
\hline Para un familiar o amigo enfermo & & & & \\
\hline Por publicidad en la T.V., radio o periódico & & & & & \\
\hline Por haber donado antes & & & & & \\
\hline Si recibo información acerca de la donación & & & & & \\
\hline Si pudiera donar aquí, en la Universidad & & & & \\
\hline
\end{tabular}

13. ¿Cree Ud. que donar sangre es dañino para la salud? $\quad$ Sí $\square \quad$ No $\square$

Sólo si respondió que "Sí", evalúe el nivel de importancia de las razones siguientes.

\begin{tabular}{|l|l|l|l|l|l|}
\hline & $\begin{array}{c}\text { Muy } \\
\text { importante }\end{array}$ & $\begin{array}{c}\text { Reguralmente } \\
\text { importante }\end{array}$ & Neutral & $\begin{array}{c}\text { No tan } \\
\text { importante }\end{array}$ & $\begin{array}{c}\text { No es importante } \\
\text { en lo absoluto }\end{array}$ \\
\hline Aumento de peso & & & & & \\
\hline Pérdida de peso & & & & & \\
\hline Anemia & & & & & \\
\hline Pérdida de la visión & & & & & \\
\hline Contagio de alguna enfermedad & & & & & \\
\hline No tener cantidad suficiente de sangre & & & & \\
\hline
\end{tabular}

Gracias por su tiempo y su colaboración 


\section{REFERENCIAS}

1. Astorga I, Amaya S. 2000. Proyecto de Regionalización de los Bancos de Sangre. Hallado en: http://www.ias-uchile.cl/files/biblioteca/ BancosdeSangre.pdf. Acceso el 3 de abril 2006.

2. Sullivan P. Developing an administrative plan for transfusion medicine-a global perspective. Transfusión. 2005(45)(4suppl):224S-40S.

3. García M, Saenz de Tejada E, Cruz J. Estudio de factores socioculturales relacionados con la donación voluntaria de sangre en la Américas. Rev Panam Salud Pública. 2003;13(2-3): 85-90.

4. Eastlund T. Monetary blood donation incentives and the risk of transfusion-transmitted infection. Transfusion. 1998;38(9):874-82.

5. Barker L, Westphal R. Voluntary, nonremunerated blood donation: still a world health goal. Transfusion. 1998;38(9):803-6.

6. Thompson W. Blood donation behavior of Hispanics in the lower Rio Grande Valley. Transfusion. 1993;33(4):333-5.

7. Ownby H, Kong F, Watanabe K. Analysis of donor return behavior. Retrovirus Epidemiology Donor Study. Transfusion. 1999;39 (10): $1128-35$.
8. Thomson R, Bethel J, Lo AY, Ownby HE, Nass CC, Williams AE. Retention of "safe" blood donors. The Retrovirus Epidemiology Donor Study. Transfusion. 1998;38(9): 359-67.

9. Mwaba K, Keikelame MJ. Blood donation behaviour and beliefs among a sample of high school students in Mmabatho. Curationis. 1995;18(3):2-3.

10. Hosain GM, Anisuzzaman M, Begur A. Knowledge and attitude towards voluntary blood donation among Dhaka University students in Bangladesh. East Afr Med J. 1997; 74(9):549-53.

11. Wiwanikit V. Knowledge about blood donation among a sample of Thai university students. Vox Sang. 2002;83(2):97-9.

12. Moore RJ. Promoting blood donation: a study of the social profile, attitudes, motivations and experience of donors. Transfus Med. 1991;1(4):201-7.

13. Fernández-Montoya A, López-Berrío A, Luna del Castillo JD. How some attitudes, beliefs and motivations of Spanish blood donors evolve over time. Vox Sang. 1998;74(3):140-7.
14. Fernández-Montoya A, de Dios Luna del Castillo J,López Berrío A, Rodríguez Fernández A. Attitudes, beliefs and motivations in blood donors and non-donors. Sangre (Barc). 1996;41(6):413-6.

15. Bhopal RS, McEwan RT, Madhok R, McCallum A. Perceptions about blood donation, transfusion and the risk of HIV infection: implications for the blood transfusion service.

16. Boulware LE, Ratner LE, Ness PM, Cooper contribution of sociodemographic, medical, and attitudinal factors to blood donation among the general public. Transfusion. 2002; 42(6):669-78

Manuscrito recibido el 20 de julio de 2006. Aceptado para publicación, tras revisión, el 18 de septiembre de 2007. AIDS Care. 1992;4(1):43-52. LA, Campbell-Lee S, LaVeist TA, et al. The

ABSTRACT Objectives. To evaluate the knowledge and attitudes of students, professors, and other staff at the University of Talca, Chile, regarding voluntary blood donation.

Methods. From June to July 2002 a previously-validated survey was administered to

\section{Blood donation: knowledge and attitudes of a university population in Chile} derstanding of blood donation, i.e., motivators and reasons for becoming a blood donor and the myths and fears that might deter blood donation. Descriptive statistics were used to analyze the data.

Results. Only $14 \%$ of survey participants had donated blood in the past, and most of these $(43.3 \%)$ were professors or other academics. Of the participants who were planning to donate blood in the future, students were the majority $(88.1 \%)$. When the males in the group that was planning to donate were compared to the females, the difference was not statistically significant $(90.0 \%$ vs. $84.2 \%$, respectively). Of the deterrents to blood donation, $73.4 \%$ said the collection instruments might not be sterile.

Conclusions. The university population represents a pool of desirable donors; an informative and motivating campaign could successfully convert the $87.3 \%$ potential donors into actual volunteer blood donors.

Key words Blood donors, universities, attitude, Chile. 